\title{
Abordaje ortodóncico preliminar de un paciente con Osteocondromatosis múltiple. Reporte de Caso
}

\author{
Preliminary orthodontic approach of a patient with multiple Osteochondromatosis. Case Report \\ Paulo Sandoval ${ }^{1, a}$, Pamela Troncoso ${ }^{1, b}$, Roxana Ferreira ${ }^{l, c}$
}

\section{RESUMEN}

La osteocondromatosis múltiple es una patología de baja prevalencia, caracterizada por la presencia de lesiones benignas formadas por cartílago hialino maduro "osteocondromas", los que se desarrollan en la metáfisis de los huesos, próximos a las placas de crecimiento.

causados por los osteocondromas incluyen malformaciones esqueléticas, acortamiento asimétrico de las extremidades, y el riesgo potencial de transformación maligna a condrosarcoma.

Reportamos el caso clínico de un paciente de 17 años de edad, diagnosticado con ésta patología a los 3,5 años de edad. Actualmente, con compromiso de la mayoría de sus articulaciones. El paciente fue derivado a la especialidad de ortodoncia para el manejo de su maloclusión dentaria. El tratamiento ideal por su maloclusion Clase III esqueletal, era ortodóncico-quirúrgico, el cual no se pudo hacer. Se realizó un tratamiento ortodóncico compensatorio que consistió en alinear los dientes realizando extracciones sin modificar sus maxilares, en espera de una cirugía ortognática al término del crecimiento.

PALABRAS CLAVE: Osteocondromatosis, osteocondroma, maloclusión, ortodoncia. (DeCS, Bireme)

\footnotetext{
${ }^{1}$ Facultad de Odontología, Universidad de la Frontera. Temuco, Chile.

a Ortodoncista. Magíster en Educación. Profesor del Departamento de Odontopediatría y Ortodoncia.

${ }^{\mathrm{b}}$ Estudiante $3^{\circ}$ año, Especialidad Ortodoncia.

c Estudiante $2^{\circ}$ año, Especialidad Ortodoncia.
} 


\section{SUMMARY}

Multiple osteochondromatosis is a disease of low prevalence, characterized by the presence of benign lesions consist mature hyaline cartilage "Osteochondromas "which develop in the metaphysis of bones near growth plates. Clinical problems caused by osteochondromas include skeletal malformations, asymmetric limb shortening, and the potential for malignant transformation to chondrosarcoma risk.

We report a case of a patient of 17 years old, diagnosed with this disease at 3.5 years. Currently, with involvement of most of its joints. The patient was referred to the specialty of orthodontics for managing your dental malocclusion. The ideal treatment for its Class III jaw was orthodontic-surgical, which could not be done. Compensatory orthodontic treatment consisting performing teeth extractions align without changing their jaws awaiting orthognathic surgery at the end of growth was performed.

\section{KEYWORDS: Osteochondromatosis, osteochondroma, malocclusion, orthodontics. (MeSH, NLM)}

\section{INTRODUCCIÓN}

La osteocondromatosis múltiple es una enfermedad generalmente hereditaria, transmitida de forma autosómica dominante. Se caracteriza por la formación anormal de cartílago debido a una mutación genética, en la cual el cartílago no se reabsorbe ni se osifica de forma habitual. En la mayoría de los pacientes pueden detectarse una historia familiar positiva y/o mutación en uno de los genes EXT (genes supresores de tumores), lo que resulta en un error en la regulación de la proliferación y maduración de condrocitos, que conduce a un crecimiento anormal de los huesos $(1,2)$.

Tiene baja prevalencia, estimándose una tasa de 0,9 a $2 / 100$ 000. Se presenta entre los 10 y 50 años de edad. Por lo general es autolimitada, y se detiene en forma espontánea cuando el paciente deja de crecer. Las características clínicas se manifiestan, generalmente, en la primera década de vida, afectando principalmente al sexo masculino en una relación de $1,5: 1(3,4,5)$.

Los osteocondromas se desarrollan como lesiones primarias solitarias o múltiples en la metáfisis ósea, en la proximidad a la placa de crecimiento del cartílago (4). Esta lesión se origina en huesos con osificación endocondral, con mayor frecuencia afecta los huesos largos, especialmente la zona distal del fémur, proximal de la tibia y proximal de húmero (1). El $40 \%$ de los tumores ocurren alrededor de la rodilla. Los huesos faciales no se ven afectados (5).

Los pacientes relatan dolor en las articulaciones afectadas, aumento de volumen y limitación en los movimientos. El diagnóstico se realiza mediante el examen físico, radiografías y biopsia. Exámenes adicionales como gammagrafía, ecografía y resonancia magnética, están indicados para la evaluación y vigilancia de las lesiones que se han convertido en sintomáticas (5).

Las masas tumorales producen deformación de los tejidos, compresiones vasculares, nerviosas y pérdida de la movilidad (5). Los huesos largos presentan menos deformaciones, pero el compromiso prematuro en el crecimiento epifisiario puede producir asimetría $\mathrm{y}$ discrepancia en la angulación y longitud de la extremidad $(2,6)$.

El manejo médico requiere de controles para observar la evolución de las lesiones. De ser necesario se realizan cirugías para su extirpación (5).

Se ha reportado que entre el $5 \%$ al $25 \%$ de los casos sufren malignificación, lo que ocurre habitualmente a partir de los 40 años de edad, la mayoría degenera en condrosarcoma periférico $(1,2)$. Esta complicación debe sospecharse si aparece dolor, crecimiento del osteocondroma después del cierre del cartílago, o hallazgos en los estudios por imágenes, dentro de los cuales las resonancia magnética es la de mayor sensibilidad $(2,4,6)$. Los pacientes con osteocondromas situados en los huesos largos o esqueleto axial, especialmente la pelvis, tienen mayor riesgo de desarrollar condrosarcoma (7). En presencia de condrosarcoma, muchas veces, se debe realizar amputación de la extremidad $(2,7)$.

Su aparición en la articulación temporomandibular (ATM) es rara, en donde sólo 75 casos han sido 
publicados, y uno con compromiso bilateral $(8,9)$. Los síntomas predominantes son dolor, inflamación, limitación de los movimientos mandibulares y crepitación (10).

Aunque en otras articulaciones, la presencia de lesiones es el doble de frecuente en los varones, en la ATM su frecuencia es cuatro veces mayor en mujeres. Al ser una enfermedad crónica y de lenta progresión, la edad media de diagnóstico en la ATM está en torno a los 55 años de edad (10).

El objetivo del presente reporte de caso fue mostrar el manejo ortodóncico compensatorio en un paciente con una patología sistémica de baja prevalencia. Además, hacer conciencia de la necesidad de un trabajo interdisciplinario en el manejo de este tipo de casos.

\section{REPORTE DE CASO}

\section{Antecedentes generales}

Paciente de género masculino de 17 años de edad, producto de tercer embarazo normal de madre de 35 años. Sin antecedentes familiares de la enfermedad. La patología debutó a los 3,5 años de edad, con un aumento de volumen en la rodilla, tras biopsia excisional de la lesión, se le diagnosticó con osteocondromatosis múltiple. A los 5 años, la enfermedad se ramificó a otras articulaciones (manos, hombros, tobillos) siendo el lado derecho

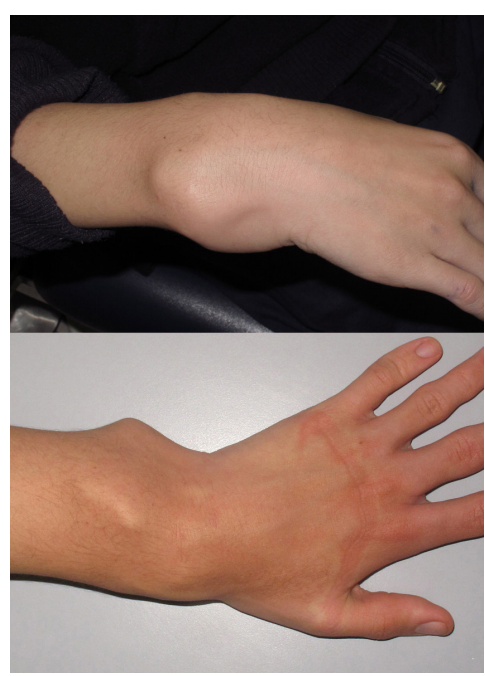

Figura 1. Arriba: Aspecto clínico del osteocondroma en la mano derecha al año 2009. Abajo: Mano izquierda al año 2013. el más comprometido al incluir también la cadera. A los 7 años se le realizó la segunda cirugía, en ésta oportunidad en la muñeca (Figura 1). Hasta la fecha se le han realizado 9 cirugías. La última, en el tobillo derecho, a los 17 años, en la cual se debió cortar el tendón calcáneo, quedando con secuelas temporales para su desplazamiento. El paciente debe usar plantillas por presentar asimetría en sus extremidades (Figura 2). No relata dolor de sus lesiones, sólo al aparecer una nueva o con el frío; para lo cual, está con indicación de Paracetamol 500 mg SOS.

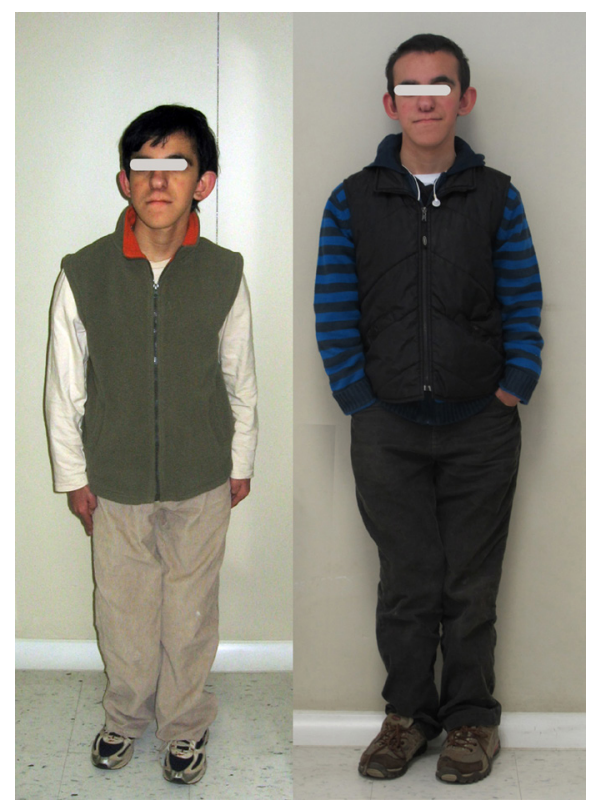

Figura 2. Aspecto físico del paciente, donde se observa su marcada asimetría.

El paciente se encuentra próximo a terminar su crecimiento, donde debería cesar la aparición de nuevos tumores.

Actualmente, sigue en controles cada 6 meses con el traumatólogo infantil y se realizan exámenes para evaluar la posible malignificación de sus lesiones.

\section{Antecedentes odontológicos}

Consulta el año 2009, a los 15 años de edad, en elárea de odontopediatría de la Universidad de la Frontera, derivado por su traumatólogo tratante, con una interconsulta que solicitaba "manejo odontológico", lo que fue muy general y llevó al paciente a recorrer varios servicios de las diferentes especialidades odontológicas antes de llegar a ortodoncia, que daba respuesta al motivo de consulta. La familia relata que era víctima de bullying en el colegio por su "doble 
corrida de dientes". Fue evaluado durante los años 2008-2009 por 2 ortodoncistas en forma particular que no lo quisieron tratar, por su patología de base.

\section{Resumen diagnóstico ortodóntico}

Paciente de género masculino, 15 años de edad, en pico puberal, con osteocondromatosis múltiple. Maloclusión clase III esqueletal. Perfil facial medio anteinclinado, dolicofacial, crecimiento horario. Dentición permanente incompleta. Discrepancia negativa maxilar y mandibular. Mesioclusión molar y canina bilateral. Mordida cruzada bilateral. Mordida invertida parcial anterior. Retroinclinación de incisivos inferiores y proinclinación de incisivos superiores.

Al exámen clínico de la ATM, el trayecto en apertura y cierre mandibular es recto. No presenta dolor, ruidos ni salto articular. La radiografía no muestra signos de presencia de lesiones en ATM (Figura 3).

En la radiografía panorámica se observa la presencia de pieza supernumeraria, primer premolar superior derecho (1.4) incluido, y segundo premolar inferior derecho (4.5) retenido (Figura 4 y 5).

\section{Plan de tratamiento}

Lo ideal en éste caso es un tratamiento ortodónticoquirúrgico el que no es posible realizar por el momento; debido a que el paciente aún se encuentra en crecimiento y por los problemas económicos de la familia. Además, debemos considerar que terminando su crecimiento, va a ser intervenido por su enfermedad de base, que es prioridad.

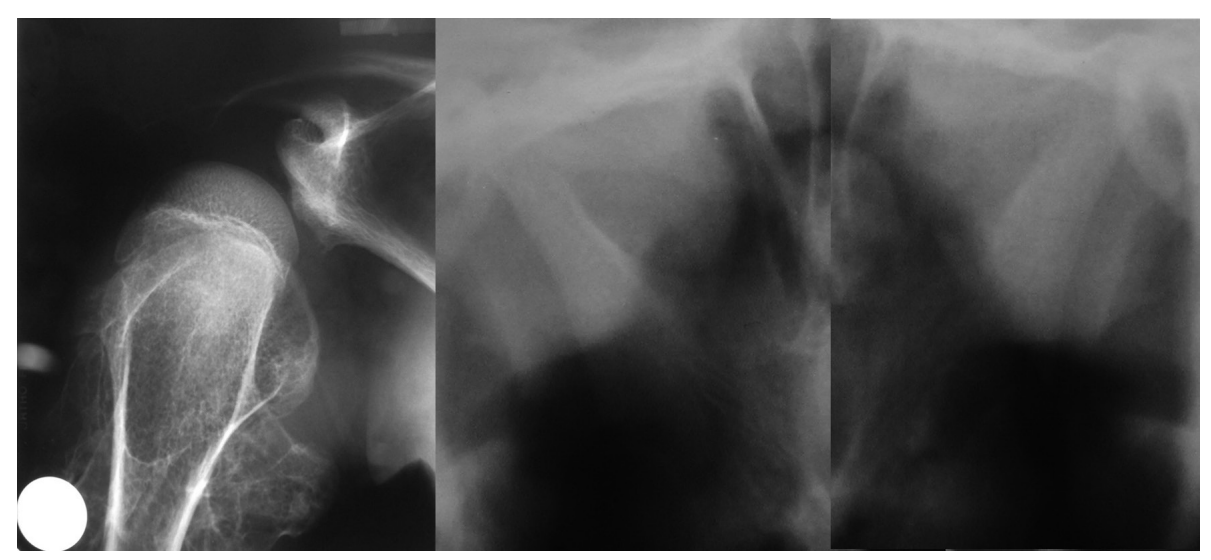

Figura 3. Exámen radiográfico donde se observa osteocondroma en hombro derecho. No se observan lesiones en ATMs.

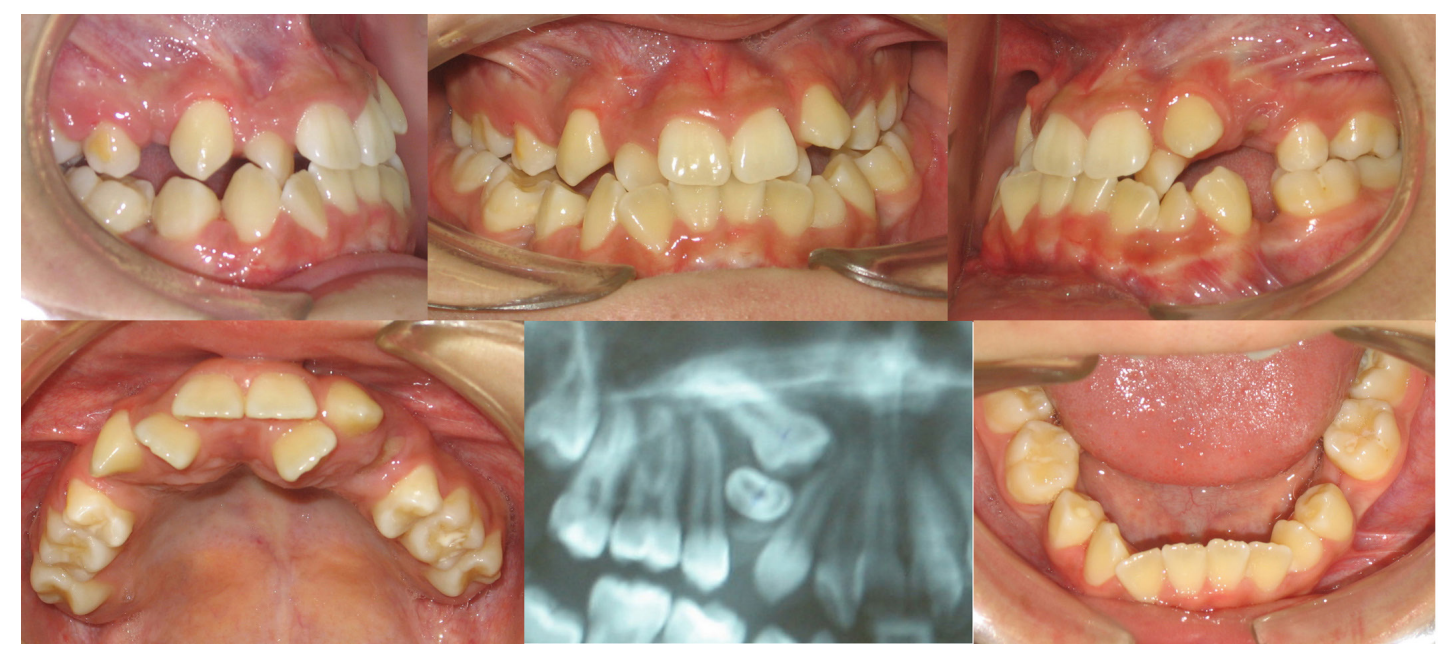

Figura 4. Aspecto intraoral al inicio del tratamiento, con un apiñamiento severo maxilar y mandibular manejado con extracciones. 


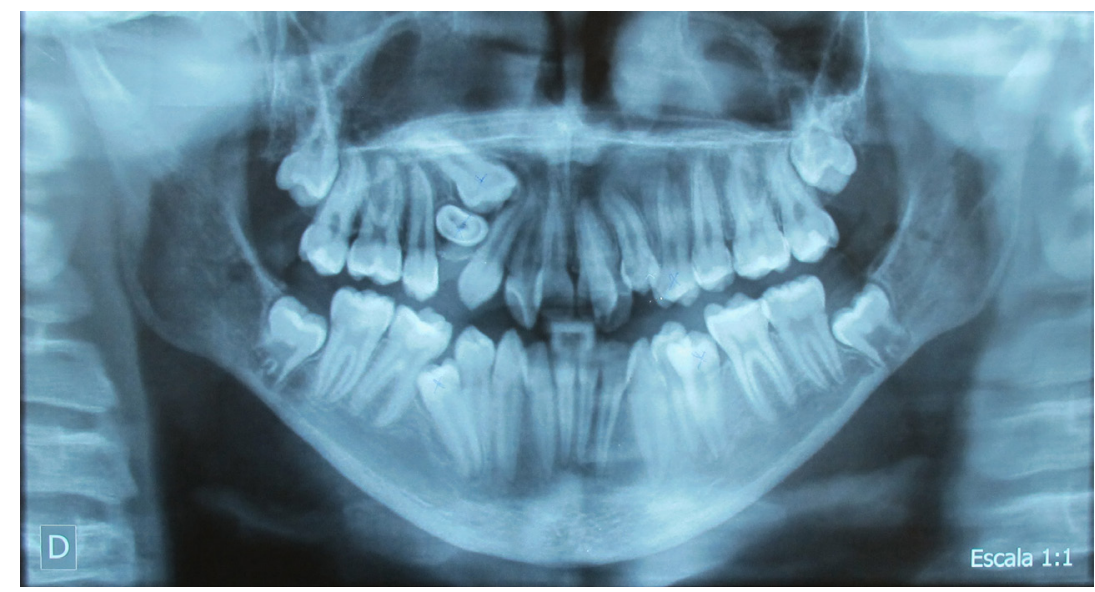

Figura 5. Radiografía panorámica inicial, donde se observa la malposición del primer premolar superior derecho por la presencia de un diente supernumerario.

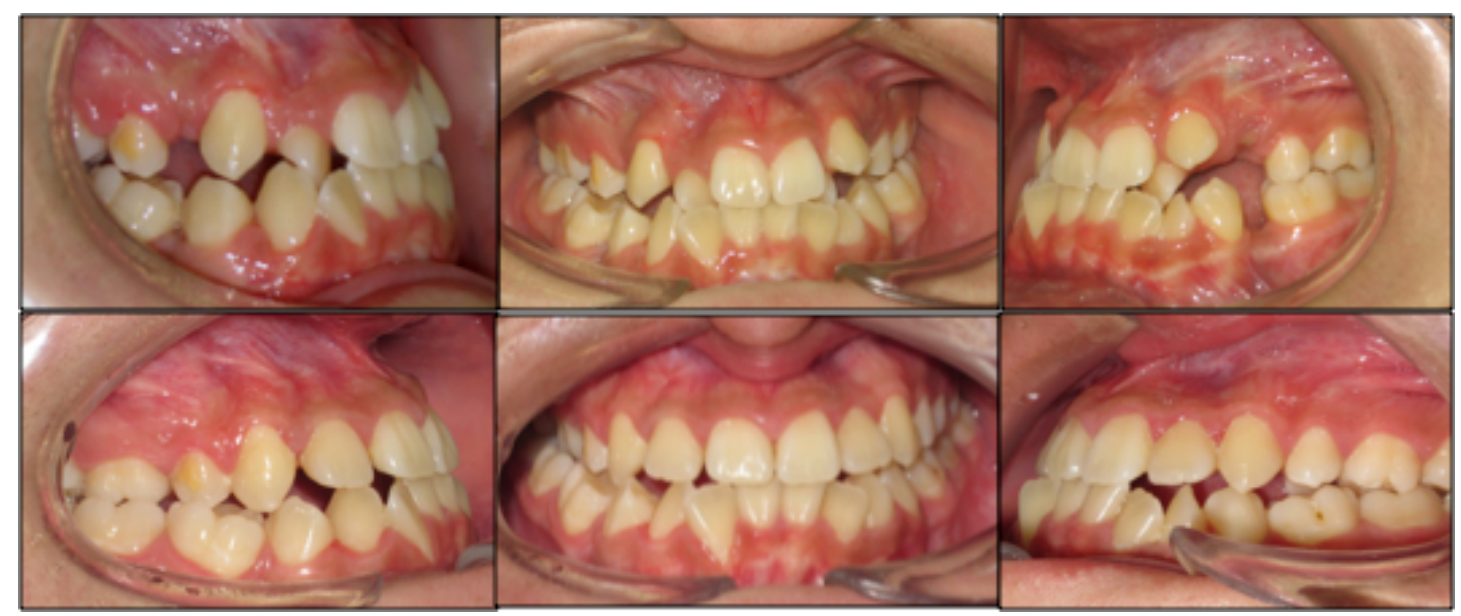

Figura 6. Vista lateral y frontal de situación inicial (superior) y final (inferior) al tratamiento con aparatos fijos en maxila.

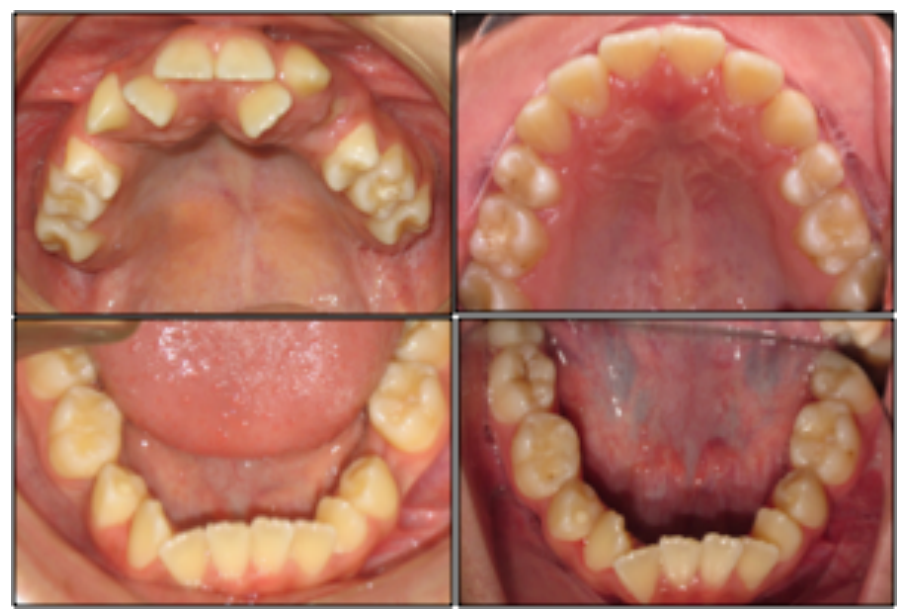

Figura 7. Vista oclusal de situación inicial (izquierda) y final (derecha) post- tratamiento. 
Por lo anterior, se opta por un tratamiento compensatorio de compromiso, en que se alinearán sus dientes para mejorar el aspecto estético y autoestima, en espera de cirugía ortognática a futuro. Los objetivos del tratamiento fueron: manejar la discrepancia dentomaxilar negativa maxilar y mandibular, satisfacer los requerimientos estéticos del paciente y su familia, mantener la compensación dentaria mandibular.

Acciones: exodoncias de primeros premolares superiores $(1.4,2.4)$ y segundos premolares inferiores $(3.5,4.5)$, necesarias para resolver la situación de apiñamiento actual, y para la futura preparación quirúrgica del paciente. Instalación de aparatología fija prescripción MBT .022 en maxila, en mandíbula no se realiza instalación (Figura 6 y 7).

\section{DISCUSION}

La osteocondromatosis múltiple generalmente es hereditaria, y se transmite en forma autosómica dominante (1). El caso expuesto no presenta antecedentes familiares de la enfermedad, por lo que podría estar dentro del $25 \%$ no hereditaria, que ocurre por mutaciones espontáneas.

Las manifestaciones clínicas se presentan generalmente en la primera década de vida, afectando principalmente al sexo masculino, lo que coincide con el caso expuesto (6).

Por las similitudes cínicas, es necesarios establecer un diagnóstico diferencial con otros tumores benignos formadores de cartílago como son la enfermedad de Ollier y el Síndrome de Maffuci, en las cuales las lesiones óseas o encondromas se presentan con predominio unilateral en el centro de huesos tubulares cortos (5), a diferencia de los osteocondromas, que son lesiones bilaterales, ubicados en la superficie ósea de huesos largos y planos $(1,11)$. Además, en el síndrome de Maffuci, los encondromas se asocian a hemangiomas (12).

Aunque la Osteocondromatosis múltiple sea una patología benigna y de rara presentación, puede tener una afección extensa con deformidades que comprometen la funcionalidad y que hacen difícil su tratamiento, lo que se convierte en un desafío al abordar un caso con éstas características (2).
Además, hay que considerar que la patología puede presentar recidivas, afectar otras articulaciones como ATMs o sufrir transformación maligna (2), lo que obliga a realizar un seguimiento a largo plazo y estar en sintonía con el resto del equipo profesional que maneja al paciente.

El riesgo de malignificación es mayor cuando las lesiones son múltiples o cuando se comprometen huesos largos o del esqueleto axial como la pelvis, ambos factores de riesgo ocurren en el caso presentado $(1,7)$ Además, en un paciente con ésta patología debemos ser cuidadosos al momento de someterlo a radiación; ya que, también constituye un riesgo de malignificación de las lesiones (6).

Lamentablemente, no se encontraron reportes que asociaran la osteocondromatosis múltiple con maloclusiones dentarias; por lo tanto, no podemos compararlo con casos similares, y aproximarnos a conocer si la desarmonía dentomaxilar que presentaba el paciente tiene alguna relación con su patología base, al parecer la única asociación sería el riesgo de formación de osteocondromas a nivel de la ATM (8). Utumi y col. (13) reportan el caso de una joven que presentó un osteocondroma en la articulación temporomandibular y que fue tratada con cirugía de resección del cóndilo unilateral, a diferencia del presente caso que requerirá una cirugía convencional (13).

Tampoco se encontraron reportes de pacientes con ésta patología tratados con ortodoncia. Sin embargo, Küçükesmen y col. (14) presentaron el caso de un niño de 11 años de edad, a quien se le realizó una prótesis pediátrica removible que reemplazó a los dientes ausentes. El niño presentaba una higiene muy deficiente (similar al presente caso), secuela de hipomineralización molar-incisiva y caries muy avanzadas a diferencia del presente reporte (14).

Si bien el tratamiento realizado no fue el ideal, era lo que estaba planificado, por lo que la falta de llaves oclusales y otros parámetros oclusales se espera lograrlos luego de la cirugía ortognática. Por tanto sólo se enfocó en resolver el apiñamiento dentario y alinear el arco superior, dando respuesta al motivo de consulta del paciente y de esta manera fortalecer el aspecto psicológico, que le ha permitido socializar en su entrono así como comenzar a estudiar una 
carrera del área de la salud. El tratamiento quirúrgico definitivo puede se demore porque la prioridad del paciente es resolver quirúrgicamente las lesiones de su patología de base.

\section{CONCLUSIONES}

El tratamiento realizado ha mejorado la calidad de vida del paciente permitiendo mejorar la estética dentaria, con un evidente aumento de su autoestima. Fue posible realizar un tratamiento ortodóncico compensatorio, en espera de una cirugía ortognática a pesar de la patología base que presentaba, la que limitó a otros ortodoncistas a brindar una atención ortodóncica oportuna.

\section{Correspondencia}

Dr. Paulo Sandoval Vidal

Dirección: Manuel Montt $1152^{\circ}$ Piso. Temuco,

Chile.

Correo electrónico: paulo.sandoval@ufrontera.cl

\section{REFERENCIAS BIBLIOGRÁFICAS}

1 Zafra A, Dominguez J, Lobato RD, Rivas JJ, et al. Condrosarcoma cervical en paciente con osteocondromatosis múltiple. Neurocirugía 1993; 4(3):233-238.

2. Carpintero P, Del Fresno JA, Carpintero R, Galvez MJ, Marín MA. Complicaciones de los osteocondromas. Revista Española de Cirugía Osteoarticular. 2009; 44(237):23-29.

3. Zak B, Crawford B, Esko J. Hereditary multiple exostoses and heparan sulfate polymerization. Biochimica et Biophysica Acta. 2002; 1573(3):346355.

4. Stieber J.R, Dormans J.P. Manifestations of hereditary multiple exostoses. J Am Acad Orthop Surg 2005; 13:(2)110-120.

5. Bovée J. Multiple osteochondromas. Orphanet Journal of Rare Diseases 2008; 3(3):1-7.
6. Murphy E, McKenzie C. Multiple osteochondromas in the archaeological record: a global review. Journal of Archaeological Science. 2010; 37(9): 2255-2264.

7. Verdegaal SH, Bovée JV, Pansuriya TC, et al. Incidence, predictive factors, and prognosis of chondrosarcoma in patients with Ollier disease and Maffucci syndrome: an international multicenter study of 161 patients. Oncologist. 2011; 16(12):1771-1779.

8. Von Lindern JJ, Theuerkauf I, Niederhagen B, Bergé S, Appel T, Reich RH. Synovial condromatosis of the temporomandibular joint: clinical, diagnostic, and histomorphologic findings. Oral Surg Oral Med Oral Pathol Oral Radiol Endod. 2002; 94(1): 31-38.

9. Keogh CF, Torreggiani WC, Munk PL. Bilateral synovial condromatosis of the temporomandibular joint. Clin Radiol. 2002; 57(9): 862.

10. Martín-Graniz R, Sánchez JJ, Jorquera M, Ortega L. Synovial chondromatosis of the temporomandibular joint: a clinical, radiological and histological study. Med Oral Patol Oral Cir Bucal. 2005; 10(3):272-276.

11. Shaheen F, Ahmad N, Gojwari T, Teli MA, Resold R, Singh M. Multiple enchondromatosis: Olliers Disease. JK Science. 2010; 12(4): 207-209.

12. Cammarata-Scalisi F, Sanchez-Flores R, StockLeytonn F, Labrador-Chacón N, Cammarata-Scalisi G. Exoxtosis múltiple hereditaria. Diagnóstico de un caso y diagnóstico diferencial de las encondromatosis. Acta Ortopédica Mexicana. 2012; 26(6):388-392.

13.Utumi RE, Pedron IG, Perrella A, Zambon CE, Ceccheti MM, Cavalcanti MGP. Osteochondroma of the temporomandibular joint: a case report. Braz Dent J 2010; 21(3): 253-258.

14. Küçükesmen Ç, Özen B, Akçam M. Multiple hereditary osteochondromatosis: a case report. Eur J Dent. 2007; 1(3): 183-187.

Recibido: 17/03/2014

Aceptado: 10/05/2014 